\title{
SOME RELATIONSHIPS INVOLVING SUBORDINATION
}

\section{R. M. BLUMENTHAL ${ }^{1}$}

If $X(t)$ is a stochastic process taking values in some space $X$ and $g$ is a real-valued function on $X$ then we may consider the process $g(X(t))$. Often from some knowledge about the process $X(t)$ and the function $g$ one can deduce properties of the process $g(X(t))$. For example: if $X(t)$ is Brownian motion in $R^{n}$ and $g$ is a subharmonic function on $R^{n}$ that doesn't grow too fast then $g(X(t))$ is a semimartingale (Doob [2, p. 92]). Sometimes the implication can be reversed in the sense that from some knowledge of the processes $X(t)$ and $g(X(t))$ one can deduce properties of the function $g$. For example: if $X(t)$ is Brownian motion in $R^{n}$ and $g(X(t))$ is a martingale then $g$ is equal almost everywhere to a harmonic function (Theorem 2, below). In this note we will consider implications of both sorts in several rather special cases, making use of the notion of subordination of one process to another.

1. The notion of subordination is systematically introduced in Bochner [1, p. 91]. We will use the following definition: let $x$ be a set and $B$ a Borel field of subsets of $x$, and let $P(t, x, B)(t \geqq 0)$ be a stationary Markov transition function on $(X, B)$. (We always set $P(0, x, B)$ equal to 1 or 0 according to whether $x$ is or is not in $B$. In addition we will assume that for each $B$ in $B$ the transition function $P$ is jointly measurable in $(t, x)$ with respect to the product Borel field $J \times B$ where $J$ denotes the Borel subsets of $[0, \infty)$ ). Further, let $G(u, A), u \geqq 0, A$ in $\mathrm{J}$, be a family of probability measures on $\Im$ such that (a) $G(u,\{0\})=1$ or 0 according to whether $u=0$ or $u>0$, (b) for every $\delta>0, G(u,[0, \delta]) \rightarrow 1$ as $u \rightarrow 0$, (c) for all $u \geqq 0$, $v \geqq 0 G(u, \circ) * G(v, \circ)=G(u+v, \circ)$ where $*$ denotes convolution. Define a function $Q(u, x, B)$ by the equation

$$
Q(u, x, B)=\int_{0}^{\infty} P(t, x, B) G(u, d t) .
$$

Using property (c) it is easy to verify that $Q$ is again a Markov transition function on $(X, B)$. It is also easy to show that for each $B$ in $B, Q$ is jointly measurable in $(u, x)$, but this is not important. If $\{X(t) ; t \geqq 0\}$ is a time homogeneous Markov process with $P$ as

Received by the editors October 10, 1958.

${ }^{1}$ Research carried out under an Office of Naval Research contract. 
transition function and $\{Y(t) ; t \geqq 0\}$ is a time homogeneous Markov process with $Q$ as transition function and with the same initial distribution as the $X$ process then we say that the $Y$ process is subordinate to the $X$ process, and we call $G$ the subordinator.

The most familiar application of this notion is to the symmetric stable processes in $R^{n}$. The symmetric stable process of index $\alpha$ $\left(0<\alpha^{\prime} \leqq 2\right)$ is a process with stationary independent increments having the transition function $P_{\alpha}(t, x, B)=\int_{B} f_{\alpha}(t, x-y) d y$ where $f_{\alpha}(t, y)$ is the continuous probability density on $R^{n}$ having the Fourier transform $\exp \left(-t|\xi|^{\alpha}\right)$. For $\alpha=2$ we have (except for a scale factor in the time parameter) the Brownian motion. If $G_{\gamma}(u, A)$ has the Laplace transform $\int_{0}^{\infty} e^{-r y} G_{\gamma}(u, d r)=\exp \left(-u y^{\gamma}\right) \quad(0<\gamma \leqq 1)$ then subordination by $G_{\gamma}$ transforms the process of index $\alpha$ into the process of index $\gamma \alpha$. The relationship between the densities is given by

$$
f_{\gamma \alpha}(u, x)=\int_{0}^{\infty} f_{\alpha}(t, x) G_{\gamma}(u, d t) .
$$

In terms of the sample functions the relationship between the original process and the subordinate process is this: suppose the Markov process $\{X(t) ; t \geqq 0\}$ with transition function $P$ is defined over some basic probability space $\Omega$. Suppose $\{T(u) ; u \geqq 0\}$ is a process defined over the same space $\Omega$ but completely independent of the $X$ process, having stationary independent increments, with $T(0) \equiv 0$, and with the distribution of $T(u)-T(v)(u \geqq v)$ being given by $G(u-v, \circ)$; (here we are of course supposing that $\Omega$ is large enough to support two such processes). Then the process $Y(u, \omega)$ $=X(T(u, \omega), \omega)$ is a Markov process with transition function $Q$ and hence is subordinate, with subordinator $G$, to the $X$ process. The above statement is somewhat loose and some regularity assumptions on the $X$ process and transition function $P$ are necessary to make it precise. But the idea is that the subordinate process is obtained by applying to the original process a certain type of optional sampling procedure. We will not use the sample function interpretation of subordination.

A real-valued function $g$ on $R^{n}$ will be called subharmonic if it is Borel measurable and if for every $x$ in $R^{n}$ and $r>0, g(x)$ $\leqq \int_{c} g(x-r y) \sigma(d y)$. (Here $C=\left\{y \in R^{n}|| y \mid=1\right\}$ and $\sigma$ is the measure distributing unit mass uniformly on $C$. The integral is to exist absolutely.) If the inequality above is replaced by equality then $g$ will be called harmonic. If $\{X(t) ; t \geqq 0\}$ is a process taking its values in $X$ and $g$ is real valued and $B$-measurable then $\{g(X(t)) ; t \geqq 0\}$ is a real-valued process. Whenever we say that $\{g(X(t)) ; t \geqq 0\}$ is a semi- 
martingale or martingale we mean that it is so relative to the family of Borel fields $\{\mathscr{F}(t)\}$, where $\mathcal{F}(s)$ is generated by all sets of the form $\{X(r) \in A\}$ with $A$ in $B$ and $r \leqq s$. If $X$ is a Markov process with transition function $P$ this is equivalent to the assertion that for every $t \geqq 0, E|g(X(t))|<\infty$ and that for every $r>0$ and $s \geqq 0$ $\int_{X} g(y) P(r, x, d y) \geqq g(x)$ for almost all $x$ in the sense of the distribution in $X$ of $X(s)$; (replace $\geqq$ by $=$ in the martingale case).

A measure $\mu$ on the Borel sets of $R^{n}\left(B\left(R^{n}\right)\right)$ will be called radially symmetric about $x$ in $R^{n}$ if $\mu(B)=\mu(T(B))$ whenever $B$ is a Borel set and $T$ is an orthogonal transformation about $x$ of $R^{n}$.

2. The first theorem is a trivial generalization of the theorem of Doob mentioned in the introduction.

TheOREM 1. Let $P(t, x, B)$ be a stationary Markov transition function on $R^{n}$ and let $\{X(t) ; t \geqq 0\}$ be a Markov process having $P$ as transition function. Suppose further that for every $t>0$ and $x$ in $R^{n}$ the measure $P(t, x, 0)$ is radially symmetric about $x$. If $g$ is subharmonic [harmonic] and $E|g(X(t))|<\infty$ for all $t \geqq 0$ then $\{g(X(t) ; t \geqq 0\}$ is a semimartingale [martingale].

Proof. First note that if $\mu$ is a probability measure on $B\left(R^{n}\right)$ and $x=\left(x_{1}, \cdots, x_{n}\right)$ is in $R^{n}$ then integration with respect to $\mu$ can be radialized about $x$ as follows: there is a probability measure $\nu_{x}$ on $J$ and a function $\sigma_{x}(r, A), r \geqq 0, A$ in $B\left(R^{n}\right)$ which is for each $A$ Borel measurable in $r$ and for each $r$ a probability measure in $A$ concentrating all its mass on the surface of the sphere with center at $x$ and radius $r$ such that if $f$ is any Borel measurable function on $R^{n}$ with $\int_{R^{n}}|f(u)| \mu(d u)<\infty$ then

$$
\int_{R^{n}} f(u) \mu(d u)=\int_{[0, \infty)} \nu_{x}(d r) \int_{R^{n}} f(y) \sigma_{x}(r, d y) .
$$

For this we simply regard the coordinate functions $\left(u_{1}, \cdots, u_{n}\right)$ as random variables with joint distribution $\mu$, and we let $R_{x}$ be the random variable $\left(\sum_{i=1}^{n}\left(u_{i}-x_{i}\right)^{2}\right)^{1 / 2}$. Then $\sigma_{x}(r, 0)$ is the (regular) conditional distribution of $\left(u_{1}, \cdots, u_{n}\right)$ given $R_{x}=r$ and $\nu_{x}$ is the distribution of $R_{x}$. If in addition $\mu$ is radially symmetric about $x$ then we may assume that for each $r, \sigma_{x}(r, 0)$ is the uniform distribution of unit mass on the surface of the sphere with center at $x$ and radius $r$. The fact that a conditional distribution with these properties may be set up follows from the discussion given in Loeve [3, p. 353]. Now the hypothesis $E \mid g(X(t) \mid<\infty$ for all $t$ implies that for any $t>0$ and $s \geqq 0$ the integral $\int_{R^{n}}|g(y)| P(t, x, d y)$ is finite for almost all $x$ 
relative to the distribution of $X(s)$, and if $g$ is subharmonic we have, for any such $x$,

$$
\begin{aligned}
\int_{R^{n}} g(y) P(t, x, d y) & =\int_{[0, \infty)} \nu_{x}(d r) \int_{R^{n}} g(y) \sigma_{x}(r, d y) \\
& =\int_{[0, \infty)} \nu_{x}(d r) \int_{C} g(x-r y) \sigma(d y) \geqq g(x)
\end{aligned}
$$

where $\nu_{x}$ and $\sigma_{x}$ are the measures appearing in the radial decomposition of $P(t, x, 0)$. If $g$ is harmonic the inequality in (4) is replaced by equality and in either case the proof is complete.

An implication of the other sort mentioned in the introduction is contained in the following theorem.

Theorem 2. Let $\{X(t) ; t \geqq 0\}$ be Brownian motion in $R^{n}$ and suppose $g$ is Borel measurable. If $\{g(X(t)) ; t \geqq 0\}$ is a martingale then $g$ is equal almost everywhere (Lebesgue measure) to a harmonic function.

Proof. Let us first remark that the conclusion of this theorem is immediate from the following formal viewpoint: if we assume that $g$ is continuous and that $g(X(t))$ is a martingale then it follows immediately that $A g=0$ where $A$ denotes the infinitesimal generator of the semi-group of transformations associated with the transition function of the process. Since for the Brownian motion $A$ can generally be identified as the ordinary Laplacean it follows that $g$ is harmonic. But here, as elsewhere in this paper where semi-group considerations seem appropriate, we have encountered difficulty in choosing a suitable domain for the semi-group of transformations and then obtaining enough information about the corresponding infinitesimal generator to make this viewpoint precise. So we proceed with the proof. By hypothesis for each $t>0 \int_{R^{n}}|g(y)| f_{2}(t, x-y) d y<\infty$ and

$$
\int_{R^{n}} g(y) f_{2}(t, x-y) d y=g(x),
$$

each assertion holding for almost all $x$, where $f_{2}(t, y)=2^{-n}(\pi t)^{-n / 2}$ $\cdot \exp \left(-|y|^{2} / 4 t\right)$. From the definition of $f_{2}$ it follows easily that the integral is finite for all $x$ and that the left side of (5) defines a continuous function of $x$. So we may as well assume that $g$ is itself continuous, and then (5) holds for all $t>0$ and $x$ in $R^{n}$. (5) can also be written as $\int_{R^{n}}(g(x-y)-g(x)) f_{2}(t, y) d y=0$, and then changing to polar coordinates we have

$$
k_{n} t^{-n / 2} \int_{0}^{\infty}\left(g_{r}(x)-g(x)\right) r^{n-1} e^{-r^{2} / 4 t} d r=0
$$


for all $t>0$ where $k_{n}$ is a positive constant and $g_{r}(x)=\int_{C} g(x-r y) \sigma(d y)$. From the uniqueness theorem for the Laplace transform and the fact that $g_{r}(x)-g(x)$ is continuous in $r$ it follows that $g_{r}(x)=g(x)$ for all $r>0$ so $g$ is harmonic.

THEOREM 3. Suppose the Markov process $\{X(t) ; t \geqq 0\}$ has transition function $P$, the Markov process $\{Y(t) ; t \geqq 0\}$ has transition function $Q$ and that $Y$ is subordinate to $X$ with subordinator $G$. If $\{g(X(t)) ; t \geqq 0\}$ is a semimartingale [martingale] and $\int_{0}^{\infty} E|g(X(t))| G(u, d t)<\infty$ for all $u \geqq 0$ then $\{g(Y(t)) ; t \geqq 0\}$ is a semimartingale [martingale].

Proof. From our joint measurability assumption on $P$ it follows that $E|g(X(t))|$ is a measurable function of $t$. Also the last hypothesis is equivalent to the assertion $E|g(Y(u))|<\infty$ for all $u \geqq 0$. Now by hypothesis for each $t>0$ and $s \geqq 0$ we have

$$
\int_{X} g(y) P(t, x, d y) \geqq g(x),
$$

the integral existing absolutely and the inequality holding for almost all $x$ relative to the distribution of $X(s)$. Let $\mu$ be the initial distribution of the processes, let $\Lambda$ be the set of points $(t, x)$ such that the integral in (7) fails to exist absolutely or the inequality fails to hold, and let $\Lambda_{t}=\{x \mid(t, x) \in \Lambda\}, \Lambda^{x}=\{t \mid(t, x) \in \Lambda\}$. Fix $u>0$ and $r \geqq 0$ and consider the measure

$$
\begin{aligned}
\theta_{r}(A) & =\int_{0}^{\infty} \int_{X} P(t, x, A) \mu(d x) G(r, d t) \\
& =\int_{\mathscr{X}} Q(r, x, A) \mu(d x)
\end{aligned}
$$

on $\Theta$, the measure $G(u, \circ)$ on $J$, and the product measure on $\Im \times B$ formed from these two. $\Lambda$ is in $J \times ß$ and for each $t \theta_{r}\left(\Lambda_{t}\right)=0$ so by Fubini's theorem for almost all $x$ relative to $\theta_{r}, G\left(u, \Lambda^{x}\right)=0$. If we take any nonexceptional $x$ and integrate each side of (7) with respect $G(u$, o) then we have

$$
\begin{aligned}
g(x) & \leqq \int_{0}^{\infty} G(u, d t) \int_{X} g(y) P(t, x, d y) \\
& =\int_{X} g(y) \int_{0}^{\infty} P(t, x, d y) G(u, d t) \\
& =\int_{X} g(y) Q(u, x, d y) .
\end{aligned}
$$


The justification for interchanging the integration orders may be obtained from the last hypothesis of Theorem 3. Inequality (8) holds for almost all $x$ relative to $\theta_{r}$, but $\theta_{r}$ is the distribution in $x$ of $Y(r)$ and so this inequality asserts that $\{g(Y(t)) ; t \geqq 0\}$ is a semimartingale.

In view of the paragraph following equation (2) Theorem 3 may be regarded as a consequence of Doob's theorems to the effect that optional sampling transforms martingales into martingales and semimartingales into semimartingales, but a good deal of discussion and possibly some additional hypotheses are needed to make this precise.

We will now reverse the martingale implication of Theorem 3 in the case of the symmetric stable processes in $R$. We first recall a few well-known facts about the transition densities $f_{\alpha} . f_{\alpha}(t, y)$ is continuous in $t>0, y$ in $R$ and strictly positive. Also if $\alpha<2$ then $\lim _{|y| \rightarrow \infty}|y|{ }^{1+\alpha} f_{\alpha}(1, y)=C(\alpha)>0$. Of course $f_{\alpha}(t, y)=t^{-1 / \alpha} f_{\alpha}\left(1, t^{-1 / \alpha} y\right)$. From these facts it follows easily that if $g$ is Borel measurable and $\alpha<2$ then $\int_{R}|g(x-y)| f_{\alpha}(t, y) d y<\infty$ for all $t>0$ and $x$ in $R$ if and only if $\int_{R}|g(y)| f_{\alpha}(1, y) d y<\infty$. Also for any such $g, \int_{R} g(x-y) f_{\alpha}(t, y) d y$ defines a continuous function of $x$. Further if for some $\alpha<2$, $\int_{R}|g(y)| f_{\alpha}(1, y) d y<\infty$ then for all $t>0, x$ in $R$ and $\beta$ in $[\alpha, 2]$ $\int_{R}|g(x-y)| f_{\beta}(t, y) d y<\infty$. We have already mentioned the subordinators $G_{\gamma}$ arising in the passage from one stable process to a subordinate one. Of course $G_{1}(u, 0)$ is the distribution putting unit mass at $\{u\}$. For $\gamma<1$ Wintner [4] gives $G_{\gamma}$ as

$$
G_{\gamma}(u, A)=\int_{A} u^{-1 / \gamma} g_{\gamma}\left(u^{-1 / \gamma} x\right) d x
$$

where $g_{\gamma}(x)=2 / \pi \int_{0}^{\infty} e^{-\lambda_{s} \gamma} \sin \mu s^{\gamma} \sin s x d s$ with $\lambda=\cos \pi \gamma / 2, \mu=\sin \pi \gamma / 2$. This integral formula is somewhat hard to handle, but for $\gamma=1 / 2$ we have $g_{1 / 2}(x)=(4 \pi)^{-1 / 2} x^{-3 / 2} e^{-1 / 4 x}$. In what follows $\left\{X_{\alpha}(t) ; t \geqq 0\right\}$ will denote the symmetric stable process of index $\alpha$ in $R$. For definiteness we will always assume that $X_{\alpha}(i) \equiv 0$.

Theorem 4. If $0<\alpha \leqq 1$ and $\left\{g\left(X_{\alpha}(t)\right) ; t \geqq 0\right\}$ is a martingale then for all $\beta\left\{g\left(X_{\beta}(t)\right) ; t \geqq 0\right\}$ is a martingale. More precisely g equals a harmonic function almost everywhere from which it follows that $g$ equals a constant almost everywhere.

PRoof. The discussion preceding this theorem guarantees that all the integrals that follow exist absolutely. Now by hypothesis for all $u>0, \int_{R} g(x-y) f_{\alpha}(u, y) d y=g(x)$ almost everywhere. The integral is a continuous function of $x$ so we may assume that $g$ is itself continuous, 
in which case the equality holds for all $u>0$ and $x$ in $R$. Using subordination we have

$$
\int_{0}^{\infty}\left(\int_{R} g(x-y) f_{2 \alpha}(t, y) d y\right) \frac{1}{(4 \pi)^{1 / 2}} \frac{1}{u^{2}}\left(\frac{t}{u^{2}}\right)^{-3 / 2} e^{-u^{2} / 4 t} d t \equiv g(x)
$$

for all $u>0$. The inner integral is a continuous function of $t$. After making a change of variable in the outer integral and using the uniqueness theorem for the Laplace transform we have that for $t>0$ and $x$ in $R \int_{R} g(x-y) f_{2 \alpha}(t, y) d y=g(x)$ or $\left\{g\left(X_{2 \alpha}(t)\right) ; t \geqq 0\right\}$ is a martingale. This argument can be iterated and leads to the conclusion

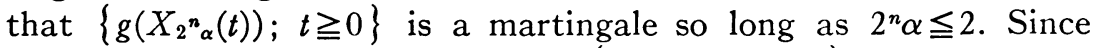
$1 \leqq 2^{n} \alpha \leqq 2$ for some $n$ it follows that $\left\{g\left(X_{\beta}(t)\right) ; t \geqq 0\right\}$ is a martingale for some $\beta$ in $[1 ; 2]$. Then by Theorem $3\left\{g\left(X_{1}(t)\right) ; t \geqq 0\right\}$ is a martingale. Using the above argument once more $\left\{g\left(X_{2}(t)\right) ; t \geqq 0\right\}$ is a martingale and by Theorem $2 g$ is then harmonic. Now $g(x)=a x+b$, but if $\alpha \leqq 1, E\left|a X_{\alpha}(1)+b\right|=\infty$ unless $a=0$, so $g$ is a constant.

Theorem 5. If $1<\alpha \leqq 2,\left\{g\left(X_{\alpha}(t)\right) ; t \geqq 0\right\}$ is a martingale, and $\int_{|y|>1}|g(y)||y|^{-(1+\delta)} d y<\infty$ for some $\delta<\alpha$ then $g$ equals a harmonic function almost everywhere.

Proof. The additional integrability hypothesis is doubtless unnecessary, but we use it to validate an interchange of integration orders in what follows. Now if $\alpha>0$ the continuous function $f_{\alpha}(t, y)$ $=t^{-1 / \alpha} f_{\alpha}\left(1, t^{-1 / \alpha} y\right)$ whose Fourier transform is $\exp \left(-t|\xi|^{\alpha}\right)$ is integrable, but it is a probability density only if $\alpha \leqq 2$. In any event the relationship $f_{\gamma \alpha}(u, y)=\int_{0}^{\infty} f_{\alpha}(t, y) G_{\gamma}(u, d t)$ between these functions continues to hold as one may easily verify. Also a few integrations by parts in the Fourier inversion formula for $f_{\alpha}$ shows that if $\alpha>2$ then $|y|^{3}\left|f_{\alpha}(1, y)\right|$ remains bounded as $y \rightarrow \infty$. Assuming now that $\alpha$ is in $(1,2) \quad(\alpha=2$ is handled by Theorem 2$)$ we have by hypothesis $\int_{R} g(x-y) f_{\alpha}(u, y) d y=g(x)$, where we may assume as usual that $g$ is continuous. If we fix $x$ then as in the proof of Theorem 4

$$
\begin{aligned}
\int_{R} g(x-y) d y \int_{0}^{\infty} f_{2 \alpha}(t, y) G_{1 / 2}(u, d t) \\
\quad=\int_{0}^{\infty}\left(\int_{R} g(x-y) f_{2 \alpha}(t, y) d y\right) G_{1 / 2}(u, d t)=g(x)
\end{aligned}
$$

subject to the legitimacy of the interchange of integration orders. That this interchange is permissible follows from the relationship (take $x=0$ and $u=1$ ) 


$$
\begin{aligned}
& \int_{0}^{\infty}\left\{\int_{|y| \leqq 1}+\int_{|y|>1}\left(\left|g(-y) t^{-1 / 2 \alpha} f_{2 \alpha}\left(1, t^{-1 / 2 \alpha} y\right)\right| d y\right)\right\} G_{1 / 2}(1, d t) \\
& \leqq \\
& \quad+\int_{R}\left|f_{2 \alpha}(1, y)\right| d y \\
& \left.\quad M \int_{|y|>1}|g(-y)| t^{-1 / 2 \alpha} B\left|t^{-1 / 2 \alpha} y\right|-(1+\delta) d y\right\} G_{1 / 2}(1, d t) \\
& =M \int_{R}\left|f_{2 \alpha}(1, y)\right| d y \\
& \quad+B \int_{0}^{\infty}\left(\int_{|y|>1}|g(-y)||y|-(1+\delta) d y\right) \frac{1}{(4 \pi)^{1 / 2}} \frac{t^{\delta / 2 \alpha}}{t^{3 / 2}} e^{-1 / 4 t} d t<\infty,
\end{aligned}
$$

where $M=\sup _{|y| \leqq 1} g(y)$ and $B$ is a positive constant. So the second double integral in (9) exists absolutely and by the uniqueness argument used in the proof of Theorem 4 it follows that for each $x$

$$
\int_{R} g(x-y) f_{2 \alpha}(t, y) d y=g(x)
$$

for almost all $t$. Integrating both sides of (10) with respect to $G_{2 / 2 \alpha}(u, d t)$ we have

$$
\begin{aligned}
\int_{0}^{\infty} & G_{2 / 2 \alpha}(u, d t) \int_{R} g(x-y) f_{2 \alpha}(t, y) d y \\
& =\int_{R} g(x-y) d y \int_{0}^{\infty} f_{2 \alpha}(t, y) G_{2 / 2 \alpha}(u, d t) \\
& =\int_{R} g(x-y) f_{2}(u, y) d y=g(x)
\end{aligned}
$$

for all $u$. The interchange of integration orders in (11) may be justified by almost exactly the same argument used to justify the previous interchange although here we need the fact that $\int_{0}^{\infty} t^{\delta / 2 \alpha} G_{2 / 2 \alpha}(u, d t)$ $<\infty$. The finiteness of this integral may be obtained by considering the Fourier transform of $G_{2 / 2 \alpha}(u, \circ)$ which is

$$
\exp \left[-u \cos (\pi \gamma / 2)|\xi|^{\gamma}(1+i(\operatorname{sgn} \xi) \tan (\pi \gamma / 2))\right], \quad \gamma=2 / 2 \alpha .
$$

The fact that $g$ is harmonic now follows from the last equality in (11) and Theorem 2, so the proof is complete.

The argument of Theorems 4 and 5 can doubtless be extended to the symmetric stable processes in $R^{n}$, the subordinators that connect the various processes being the same regardless of the dimension, 
but some justification for the existence of certain expectations and for the interchange of certain integration orders must be supplied. There is no difficulty in making the extension to $R^{n}$ for the cases $0<\alpha \leqq 1$, but we have not made the necessary verifications for the cases $1<\alpha<2$.

4. The method of subordination may be used to obtain uniqueness theorems for certain classes of transforms, and the following theorem is of this sort. $g$ denotes a Borel measurable real valued function on $[0, \infty)$ and $f_{\alpha}(t, y)$ is the continuous real valued probability density on $(-\infty, \infty)$ with Fourier transform $\exp \left(-t|\xi|^{\alpha}\right),(0<\alpha \leqq 2)$.

Theorem 6. (i) $(0<\alpha \leqq 1)$. If for every $t>0 \int_{0}^{\infty}|g(y)| f_{\alpha}(t, y) d y<\infty$ and $\int_{0}^{\infty} g(y) f_{\alpha}(t, y) d y=0$ then $g=0$ almost everywhere.

(ii) $(1<\alpha<2)$. Suppose $\sup _{|y| \leq 1}|g(y)|<\infty$. If for every $t>0$ $\int_{0}^{\infty} g(y) f_{\alpha}(t, y) d y=0$ and $\int_{1}^{\infty}|g(y)||y|-1-\delta d y<\infty$ for some $\delta<\alpha$ then $g=0$ almost everywhere.

Proof. In either case the arguments used in the proofs of Theorems 4 and 5 imply that, for every $t>0, \int_{0}^{\infty}|g(y)| f_{2}(t, y) d y<\infty$ and $\int_{0}^{\infty} g(y) f_{2}(t, y) d y=0$ and the conclusion then follows from the uniqueness theorem for the Laplace transform.

\section{REFERENCES}

1. S. Bochner, Harmonic analysis and the theory of probability, University of California Press, 1955.

2. J. L. Doob, Semimartingales and subharmonic functions, Trans. Amer. Math. Soc. vol. 77 (1954) p. 86.

3. M. Loève, Probability theory, Van Nostrand, 1955.

4. A. Wintner, Cauchy's stable distributions and an explicit formula of Mellin, Amer. J. Math. vol. 78 (1956) p. 819.

UNIVERSITY OF WASHINGTON 